\title{
Casuística de uma unidade de Ortogeriatria
}

\author{
Casuistry of an Orthogeriatrics unit
}

\author{
Mónica Brinquinho' ${ }^{1}$ Fátima Seabra ${ }^{1}$, Gonçalo Sarmento ${ }^{1}$, Rafaela Veríssimo², Agripino Oliveira ${ }^{3}$ \\ ${ }^{1}$ Interno de Formação Específica de Medicina Interna; Centro Hospitalar Vila Nova Gaia/Espinho. ${ }^{2}$ Assistente Hospitalar de Medicina Interna, Centro \\ Hospitalar Vila Nova Gaia/Espinho. ${ }^{3}$ Assistente Hospitalar Graduado de Medicina Interna, Centro Hospitalar Vila Nova Gaia/Espinho.
}

\begin{abstract}
RESUMO
A fratura do fémur está associada a importante morbimortalidade, sendo que menos de metade dos doentes regressam ao seu nível prévio de autonomia. Uma abordagem multidisciplinar permite uma reabilitação funcional imediata, bem como melhor gestão de comorbilidades, prevenção de fraturas secundárias e das quedas. Objetivos: apresentação da casuística de 3 meses de uma unidade de Ortogeriatria. Material e métodos: Descreve-se a demografia dos doentes admitidos, grau de dependência funcional, comorbilidades, intercorrências, tempo de internamento, mortalidade e destino à data de alta. 0 ganho de funcionalidade no internamento foi também avaliado. Resultados: De outubro/2015 a janeiro/2016 foram admitidos 66 doentes após cirurgia de fratura proximal do fémur. Média de idades foi 82,94 $\pm 6,93$ anos; $86 \%$ eram mulheres. 0 tempo de internamento foi de 7,8 $8 \pm 4,697$ dias, sendo 0 tempo médio para realizar cirurgia de $3 \pm 3,374$ dias. Quanto ao grau de funcionalidade prévio $47 \%$ eram Katz $\mathrm{A}$; à admissão $85 \%$ tinham mRankin $\leq 3$. Identificaram-se várias comorbilidades em $89 \%$ dos doentes; nas intercorrências, a mais frequente foi a anemia (53\%). Houve um ganho na funcionalidade relativamente à admissão na unidade de 75,8\% e em relação ao estado funcional anterior ao evento de $19,7 \%$. Setenta e um porcento dos doentes tiveram alta para o domicílio; a taxa de mortalidade foi de 1,52\%. Conclusões: A unidade demonstrou eficiência no controlo das comorbilidades, reduzidas complicações e baixa mortalidade. Registou-se um ganho funcional comparando com a admissão em 3 em cada 4 doentes e em relação ao estado basal prévio em 1 em cada 5 doentes.
\end{abstract}

Palavras-chave: fratura fémur, ortogeriatria, geriatria, reabilitação

\section{INTRODUÇÃO}

A fratura do colo do fémur é frequente nos indivíduos idosos, sendo uma das complicações mais graves da osteoporose. ${ }^{1} \dot{E}$ mais comum em mulheres, estimando-se que uma em cada três mulheres com mais de 80 anos irá sofrer uma fratura do colo do fémur ao longo da vida². Estão geralmente associadas a traumatismos de baixa frequência e 0 tratamento de primeira linha é cirúrgico. ${ }^{1,3}$

Estão descritas taxa de mortalidade variáveis em fase aguda (5 a 10\%) $)^{1,4}$. Ao fim de um ano estima-se que 10 a $20 \%$ destes doentes possam falecer, metade apresenta alguma perda funcional e apenas $30 \%$ dos doentes tenham recuperado a sua capacidade funcional para os níveis prévios aos da ocorrência da fratura ${ }^{3}$

No sentido de otimizar o tratamento e reabilitação dos doentes com fratura do fémur foram criadas unidades de Ortogeriatria. Estas unidades funcionais permitem uma abordagem multidisciplinar orientada para a reabilitação funcional imediata, bem como para a gestão de comorbilidades, prevenção de fraturas secundárias e das quedas ${ }^{1,3,4,5}$. Associam-se a redução

\section{ABSTRACT}

Hip fracture is associated with important morbimortality; less than half of the patients go back to the same functional degree. A multidisciplinary approach can enable immediate functional rehabilitation, better treatment of comorbidities and prevention of secondary fractures and falls. Objectives: to describe the casuistry of an Orthogeriatrics unit in a three month period. Methods: Description of demographic variables, previous degree of dependency, comorbidities, medical or surgical occurrences, mortality and destination at discharge. The functional gain was also evaluated. Results. From October/2015 through January/2016, 66 patients were admitted to the unit, after surgical correction of proximal hip fracture. The mean age at admission was $82,94 \pm 6,93$ years; $86 \%$ were women. The mean length of stay in hospital was $7,8 \pm 4,68$ days; in average the patients waited $3 \pm 3,37$ days to surgery. Forty-seven percent of patients were Katz A on admission; $85 \%$ had mRankin scale $\leq 3$. Various comorbidities were identified in $89 \%$ of patients; the most frequent medical occurrence was anemia (53\%). There was a gain in function in $75,8 \%$ of patients considering the time of admission and in 19,7\% of patients compared to the previous functional status. Seventy-one percent of patients were discharged home; the mortality rate was $1,52 \%$. Discussion: The Orthogeriatrics unit was efficient in controlling comorbidities, and showed a low rate of complications and mortality. Three in every four patients showed functional improvement, comparing to time of admission and 1 in every five ptients comparin with previous function.

Keywords: Hip fracture, Orthogeriatrics, Geriatrics, Rehabilitation

das complicações, a maior taxa de cirurgia precoce, redução da mortalidade, melhoria funcional à alta e a longo prazo e diminuição do tempo de internamento. ${ }^{1,6-9}$

A unidade de Ortogeriatria do Centro Hospitalar de Vila Nova de Gaia/ Espinho foi criada a 18 de Outubro de 2015, com 0 objetivo de tratar os doentes que tenham uma fratura do fémur, de modo a gerir as complicações médicas, reabilitar e reinserir o doente 0 melhor e o mais precocemente possível na sua comunidade.

Os autores pretendem com este trabalho descrever a casuística da unidade, relativa aos seus 3 primeiros meses de existência.

\section{MATERIAL E MÉTODOS}

Análise retrospetiva dos doentes admitidos na unidade de Ortogeriatria no período de 18 de Outubro de 2015 a 31 de Janeiro de 2016. Caraterizaram-se as seguintes variáveis: dados demográficos (sexo e idade), grau de dependência funcional em três momentos: prévio, à admissão e à data de alta da unidade; comorbilidades, intercorrências, tempo de internamento e de espera cirúrgica, mortalidade e destino à data de alta. 
Para avaliação do grau de dependência funcional foram utilizadas as escalas de Katz e de Rankin modificada.

Na análise estatística utilizaram-se técnicas estatísticas descritivas de tendência central e o teste não paramétrico de Wilcoxon. Os resultados foram considerados estatisticamente significativos para um intervalo de confiança de $95 \%(p<0,05)$.

\section{RESULTADOS}

No período de três meses a que se refere este trabalho foram admitidos na unidade de Ortogeriatria 66 doentes, com média de idade de 82,9 anos; $25 \%$ dos doentes tinha mais de 87 anos. A maioria dos doentes (86\%) era do sexo feminino (Tabela 1).

A média de dias de internamento na unidade foi de 7,8. 0 tempo médio de espera até cirurgia foi de 72 horas, embora 40 doentes tenham sido operados nas primeiras 48h (Tabela 1). Identificaram-se múltiplas comorbilidades na população admitida na unidade no período referido - em média 3 comorbilidades por doente; $89 \%$ dos doentes referiam pelo menos uma comorbilidade. A hipertensão arterial foi a mais frequentemente registada (47 doentes). (Tabela 2). No que diz respeito às intercorrências, a anemia foi a mais frequente, tendo sido registada em 61 doentes (Tabela 2). Apenas 21 doentes necessitaram de suporte transfusional com glóbulos rubros. À data de alta todos os doentes foram orientados para consulta de Ortogeriatria que inclui avaliação por Medicina Interna, Ortopedia e Medicina Física e da Reabilitação. Quarenta e sete doentes tiveram alta direta para 0 domicílio, 10 foram orientados para reabilitação em unidade de cuidados continuados, 2 para o Centro de Reabilitação do Norte e 1 para Lar.
Tabela 1. Caraterização dos doentes admitidos

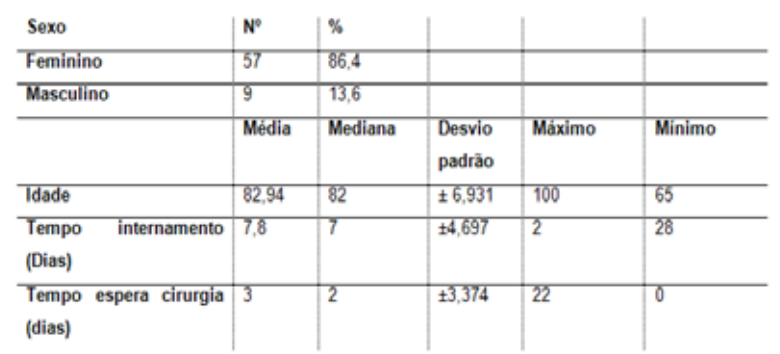

Foram transferidos 5 doentes para o internamento da Medicina Interna por apresentarem intercorrências que o justificaram. Foi registado um óbito - taxa de mortalidade de 1,52. (Tabela 3) No que diz respeito à avaliação funcional e considerando a escala de Katz, a maioria dos doentes (31 doentes, 47\%) era independente para a realização de atividades de vida diária (KatzA); 17 doentes (26\%) eram Katz B, 9 doentes (14\%) eram Katz C, 4 doentes (6\%) eram Katz D, 4 doentes (6\%) eram Katz E e 1 doente (1\%) era Katz F. Já no que diz respeito à escala de Rankin modificada (mRankin) para avaliação funcional, previamente à admissão na unidade $83 \%$ doentes tinham mRankin<3, à admissão na unidade 53\% tinham mRankin de 5 e à data de alta 73\% tinham mRankin 1-3 (gráfico 1).

Foi efetuada uma avaliação do ganho de funcionalidade comparando o valor de mRankin à alta com dois momentos distintos: à admissão - verificou-se um ganho de funcionalidade em $75 \%$ dos doentes, e ao estado prévio à admissão - houve ganho de função em 19,8\% dos doentes.

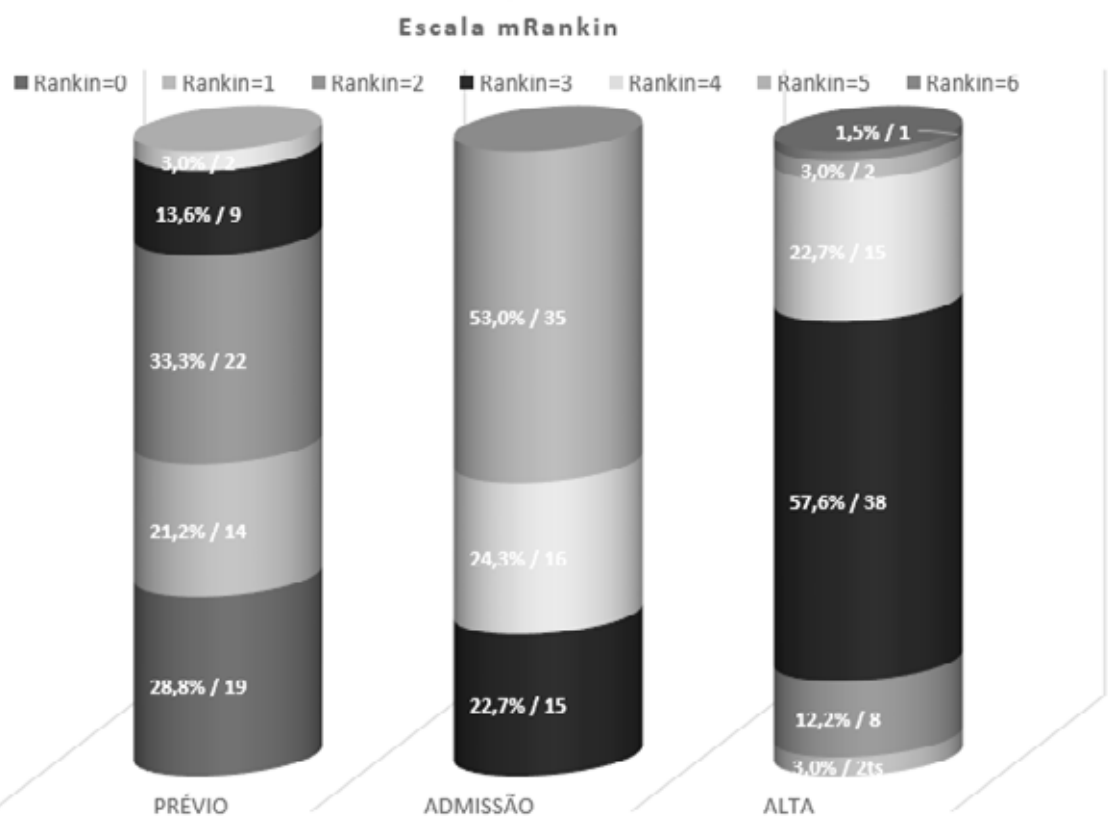


Tabela 2. Comorbilidades e intercorrências

\begin{tabular}{l|cc}
\multicolumn{3}{c}{ Comorbilidades } \\
& & \\
\cline { 2 - 3 } No & $\%$ \\
\cline { 2 - 4 } Hipertensão arterial & 47 & 27 \\
\hline Insuficiência cardiaca congestiva & 21 & 12 \\
\hline Diabetes mellitus & 20 & 12 \\
\hline Dislipidemia & 18 & 10 \\
\hline Fibrilação auricular & 16 & 9,2 \\
\hline Doença vascular cerebral & 14 & 8,1 \\
\hline Osteoporose & 10 & 5,8 \\
\hline Insuficiência Venosa Periférica & 8 & 4,6 \\
\hline Bócio multinodular & 7 & 4 \\
\hline Insuficiência respiratória crónica & 5 & 2,9 \\
\hline Doença de Parkinson & 4 & 2,3 \\
\hline Neoplasia & 3 & 1,7 \\
\hline \multicolumn{1}{c}{ Total } & 173 & 100 \\
\hline
\end{tabular}
\&

\begin{tabular}{lccc}
\multicolumn{3}{c}{ Intercorrências } & \\
& & $\mathrm{N}^{\circ}$ & $\%$ \\
\cline { 2 - 4 } Anemia & 61 & 53 \\
\hline Cistite & 22 & 20 \\
\hline Distúrbios hidroeletroliticos & 8 & 7,1 \\
\hline Insuficiência respiratória & 6 & 5,3 \\
\hline Insuficiência renal & 6 & 5,3 \\
\hline Delirium & 6 & 5,3 \\
\hline Insuficiência cardiaca descompensada & 5 & 4,5 \\
\hline \multicolumn{1}{c}{$\quad$ Total } & 114 & 100 \\
\hline
\end{tabular}

\section{DISCUSSÃO}

A literatura refere que a fratura do colo do fémur é particularmente frequente em mulheres idosas, com múltiplas comorbilidades $2,3,10,11$, factos comuns à amostra em análise - 86\% dos doentes eram do sexo feminino, com média de idade de 82 anos e tendo sido registada pelo menos uma comorbilidade em $89 \%$ dos doentes. Obteve-se uma reduzida taxa de intercorrências sendo as mais frequentes a anemia e a infeção do trato urinário. Apenas 31,8\% foram transfundidos com glóbulos rubros.

Em relação ao tempo de espera cirúrgico, apesar de se obter uma média de 3 dias até cirurgia, $60 \%$ dos doentes foi operado nas primeiras $48 \mathrm{~h}$, conforme recomendado na literatura ${ }^{11}$. Já no que diz respeito ao tempo médio de internamento obteve-se uma média de 7,8 dias, inferior à média descrita por JJ González-Montalvo e colaboradores ${ }^{11}$.

A taxa de mortalidade obtida nesta amostra foi $1,52 \%$, inferior à descrita na generalidade das referências bibliográficas e em particular quando comparada com a taxa de mortalidade de $7,1 \%$ obtida em unidade de traumatologia com apoio de geriatra. $^{12}$

Conforme referido, a maior parte dos doentes admitidos na unidade era autónomo, tendo-se verificado um aumento do grau de dependência à admissão na unidade, ou seja no momento do evento. A unidade demonstrou proporcionar um ganho de funcionalidade em relação ao momento da admissão em 3 em cada 4 doentes, e em relação ao estado prévio em 1 em cada 5 doentes, cumprindo assim um dos principais pressupostos para a criação destas unidades - 0 da reabilitação imediata ${ }^{1}$.

Tabela 3. Destino à data de alta

\begin{tabular}{lccc} 
& Destino & № & $\%$ \\
\hline Domicílio & & 47 & 71,2 \\
\hline Rede Nacional de Cuidados Continuados & 10 & 15,2 \\
\hline Medicina & & 5 & 7,58 \\
\hline Centro de Reabilitação do Norte & 2 & 3,03 \\
\hline Lar & & 1 & 1,52 \\
\hline Óbito & 1 & 1,52 \\
\hline & TOTAL & 66 & 100
\end{tabular}

\section{CONCLUSÃO}

Este trabalho corresponde a uma primeira avaliação da unidade de Ortogeriatria do Centro Hospitalar Vila Nova de Gaia/ Espinho, tendo já demonstrado ser eficiente no controlo das comobilidades, associar-se a baixa percentagem de intercorrências e mortalidade e a ganho de funcionalidade. Para 0 futuro pretende-se perpetuar estes bons resultados no internamento e perceber se este ganho de funcionalidade se irá manter em ambulatório.

\section{BIBLIOGRAFÍA}

1. González-Montalvo Jl, Alarcón T, Rodil BP, Pérez PG, Álvarez de Linera JLM, Garay EG. Ortogeriatria en pacientes agudos (I). Aspectos assistenciales. Ver Esp Gerontol. 2008; 43 (4): 239-251.

2. Santos CIRR. Os custos das fraturas de etiologia osteoporótica em mulheres: institucionalização na Rede Nacional de Cuidados Continuados (RNCCl) e lares de $3^{a}$ idade.2010

3. Branco J, Felicíssimo P, Monteiro J. A epidemiologia e o impacto socioeconómico das fraturas da extremidade proximal do fémur. Uma reflexão sobre o padrão atual de tratamento da osteoporose grave. 2009.

4. Roche JJ, Wenn RT, Sahota 0, Moran CG. Effect of comorbidities and postoperative complications on mortality after hip fracture in elderly people: prospective observational cohort study. BMJ. 2005;331(7529):1374.

5. S.P. Teo, J. Mador / Orthogeriatrics service for hip fracture patients in Dunedin Hospital: Achieving standards of hip fracture care.Journal of Clinical Gerontology \& Geriatrics 3 (2012) $62 \mathrm{e} 67$

6. Khasraghi FA, Christmas C, Lee EJ, Mears SC, Wenz JF. Effectiveness of a multidisciplinary team approach to hip fracture management. J Surg Orthop Adv. 2005:14:27-

7. Stenvall M, Olofsson B, Nyberg L, Lundström M, Gustafson Y. Improved performance in activities of daily living and mobility after a multidisciplinary postoperative rehabilitation in older people with femoral neck fracture: a randomized controlled trial with 1 year followup.J Rehabil Med. 2007;39:232-8

8. Antonelli Incalzi R, Gemma A, Capparella 0, Bernabei R, Sanguinetti C, Carbonin PU. Continuous geriatric care in orthopedic wards: A valuable alternative to orthogeriatrics units. Aging Clin Exp Res.1993;5:207-16

9. Álvarez Nebreda ML, Marañón Hernández E, Alonso Armesto M, Toledano Iglesias M, Vidán Astiz MT, García Alhambra MA, et al. Eficacia de una unidad de ortogeriatría en el tratamiento integral del anciano con fractura de cadera: comparación con un modelo previo.Rev Esp Geriatr Gerontol. 2005:40 Supl 1:52.

10. National Clinical Guideline Centre. The management of hip fracture in adults. London: National Clinical Guideline Centre; 2011

11. Hudson H, Hepherd R, Ruckledge A, Ajaj A. Orthogeriatrics. GM 2013

12. J.I. González Montalvo et al. La unidad de ortogeriatría de agudos. Evaluación de su efecto en el curso clínico de los pacientes con fractura de cadera y estimación de su impacto económico. Rev Esp Geriatr Gerontol. 2011:46(4):193-199. 